Research Paper

\title{
Triptolide Attenuates Renal Tubular Epithelial-mesenchymal Transition Via the MiR-188-5p-mediated PI3K/AKT Pathway in Diabetic Kidney Disease
}

\author{
Mei Xue, Ying Cheng, Fei Han, Yunpeng Chang, Yang Yang, Xiaoyu Li, Li Chen, Yunhong Lu, Bei Sun ${ }^{\bowtie}$, \\ Liming Chen ${ }^{凶}$
}

Key Laboratory of Hormones and Development (Ministry of Health), Tianjin Key Laboratory of Metabolic Diseases, Tianjin Metabolic Diseases Hospital \& Tianjin Institute of Endocrinology, Tianjin Medical University

$\triangle$ Corresponding authors: Liming Chen, MD, PhD. E-mail: xfx22081@vip.163.com. Tel: +86-22-23333266; Bei Sun, MD, PhD. E-mail: beisun@tmu.edu.cn. Tel: +86-22-23542602. Address: Key Laboratory of Hormones and Development (Ministry of Health), Tianjin Key Laboratory of Metabolic Diseases, Tianjin Metabolic Diseases Hospital \& Tianjin Institute of Endocrinology, Tianjin Medical University, 300070 Tianjin, China. Fax: +86-22-23528460

(c) Ivyspring International Publisher. This is an open access article distributed under the terms of the Creative Commons Attribution (CC BY-NC) license (https://creativecommons.org/licenses/by-nc/4.0/). See http://ivyspring.com/terms for full terms and conditions.

Received: 2017.11.26; Accepted: 2018.02.16; Published: 2018.09.07

\begin{abstract}
Triptolide possesses the trait of renal protection. Epithelial-mesenchymal transition (EMT) is closely linked to the pathogenesis of diabetic kidney disease (DKD). MicroRNAs have recently emerged as critical regulators of DKD. However, it is poorly understood whether triptolide alleviates renal EMT by regulating microRNAs in DKD. In this study, we found that triptolide decreased albuminuria, improved the renal structure and reduced renal EMT in rats with DKD. Furthermore, activation of the PI3K/AKT signaling pathway was increased in diabetic rats, which was partly reversed by triptolide. Triptolide also alleviated glucose-induced EMT in HK-2 cells in vitro. PI3K/AKT signaling pathway activation was reduced after triptolide treatment. Moreover, triptolide decreased the increase in miR-188-5p expression stimulated by high glucose levels in HK-2 cells. miR-188-5p inhibited PTEN expression by directly interacting with the PTEN 3'-untranslated region. Additionally, downregulation of miR-188-5p, which imitates the effects of triptolide, attenuated the activation of the PI3K/AKT pathway and HG-induced EMT, whereas miR-188-5p overexpression reversed the effects of triptolide on the PI3K/AKT pathway and EMT. In conclusion, we demonstrated that triptolide ameliorates renal EMT via the PI3K/AKT signaling pathway through the interaction between miR-188-5p and PTEN, indicating that miR-188-5p may be a therapeutic target of triptolide in DKD.
\end{abstract}

Key words: Triptolide, Epithelial-mesenchymal transition, MiR-188-5p, PTEN, Diabetic kidney disease.

\section{Introduction}

Diabetic kidney disease (DKD) is one of the most severe microvascular complications of diabetes mellitus and has become the leading cause of end-stage renal disease worldwide [1]. According to the latest study, the estimated overall prevalence of diabetes and pre-diabetes among adults in China is $10.9 \%$ and $35.7 \%$, respectively [2]. Because of the increasing prevalence of diabetes, 24.3 million patients suffer from DKD and chronic kidney disease related to diabetes, and these diseases have become more common than chronic kidney disease related to glomerulonephritis in China [3]. Although tight control of blood glucose and blood pressure levels is helpful to prevent the deterioration of renal function, the growing incidence of DKD requires more therapeutic agents based on the molecular mechanisms of DKD [4].

Multiple pathological changes result in progressive impairment of renal function during DKD, including accumulation of extracellular matrix, glomerulosclerosis and tubulointerstitial fibrosis [5, 6]. Many lines of evidence support a pathogenic role 
for renal tubular injury in DKD [7]. Renal epithelial-mesenchymal transition (EMT) is a process whereby renal tubular cells undergo a gradual transformation from an epithelial phenotype to a mesenchymal phenotype, during which cells lose epithelial features, such as reduced E-cadherin expression, and gain mesenchymal features, such as increased vimentin and a-SMA expression $[8,9]$. The relationship between EMT and renal fibrosis has been reviewed [9]. Recently, EMT has been recognized as an essential pathogenic process for the development of tubulointerstitial fibrosis in DKD [10].

MicroRNAs (miRNAs) are small non-coding RNAs that directly interact with the $3^{\prime}$-untranslated region ( $3^{\prime}$-UTR) of their target messenger RNAs (mRNAs) to regulate gene expression by translational inhibition or degradation of target mRNAs. It is estimated that $60 \%$ of human protein-encoding genes are targeted by miRNAs that participate in regulating important physiological functions; hence, miRNAs have been reported to be involved in various diseases [11]. Accumulating evidence indicates that miRNAs play important roles in DKD and have become important targets for therapeutic interventions $[6,10$, 12]. Increasing evidence also supports a pivotal role for the phosphatidylinositol 3-kinase/protein kinase $\mathrm{B}$ (PI3K/AKT) signaling pathway in regulating EMT in multiple diseases [13, 14], including DKD [15]. Phosphatase and tensin homolog (PTEN), a tumor suppressor, has the opposite activity of PI3K and negatively regulates PI3K-mediated signaling pathways [16]. Recent studies have shown that PTEN is involved in EMT and that PTEN expression is influenced by many miRNAs [17, 18]. However, it remains unclear whether PTEN and the PI3K/AKT signaling pathway are involved in miRNA-induced EMT in DKD.

Tripterygium wilfordii Hook F (TwHF), a traditional Chinese herbal plant, has been clinically applied to treat DKD for several years due to its immunosuppressive and anti-inflammatory properties [19, 20]. Our laboratory has previously demonstrated that the TwHF extract alleviated albuminuria levels, glomerulosclerosis, tubulointerstitial fibrosis, and renal inflammation in rats with DKD [21]. Triptolide is a pharmacologically active ingredient that is extracted from the root of TwHF. Other studies have also reported that triptolide dramatically attenuates renal injury and regulates immune-inflammatory responses in DKD animal models [22-24]. In an in vitro study, researchers found that triptolide inhibited glomerular mesangial cell proliferation [25]. However, whether triptolide is associated with renal EMT during DKD is still unknown.
In this study, we explored the mechanism by which triptolide improves EMT in rats with DKD and human proximal tubular epithelial (HK-2) cells. We demonstrated that miR-188-5p enhanced EMT by promoting the PI3K/AKT signaling pathway by targeting PTEN and that triptolide ameliorated EMT by regulating the expression of miR-188-5p in DKD.

\section{Results}

\section{Effects of triptolide on the physical and biochemical parameters in different groups}

Compared with the NC group, body weight was significantly reduced, while blood glucose, TG and TC were elevated in both the DKD and DKD+TP groups. We also observed that triptolide caused no differences in the body weight, blood glucose and blood lipid profiles between DKD and DKD+TP rats (Table 1). The kidney weight to body weight ratio $(\mathrm{KW} / \mathrm{BW})$ and UMA levels were significantly increased in the DKD group compared with the NC group, which was markedly alleviated after triptolide treatment (Table 1). Furthermore, no significant changes in the ALT, AST, BUN and Scr levels were observed among the three groups, indicating that there was no notable toxicity due to triptolide in the diabetic rats (Table 1).

Table 1. Physical and biochemical parameters of experimental rats.

\begin{tabular}{llll}
\hline & NC & DKD & DKD+TP \\
\hline BW $(\mathrm{g})$ & $512.7 \pm 58.3$ & $326.8 \pm 41.6^{*}$ & $350.1 \pm 34.9^{*}$ \\
FPG $(\mathrm{mmol} / \mathrm{L})$ & $6.9 \pm 0.7$ & $27.2 \pm 3.3^{*}$ & $25.4 \pm 4.2^{*}$ \\
$\mathrm{KW} / \mathrm{BW}(\mathrm{mg} / \mathrm{g})$ & $3.02 \pm 0.21$ & $5.85 \pm 0.49^{*}$ & $4.77 \pm 0.42^{*} \#$ \\
$\mathrm{UMA}(\mu \mathrm{g} / 24 \mathrm{~h})$ & $26.5 \pm 9.2$ & $243.6 \pm 35.1^{*}$ & $159.0 \pm 29.3^{*} \#$ \\
$\mathrm{TG}(\mathrm{mmol} / \mathrm{L})$ & $1.6 \pm 0.9$ & $4.1 \pm 1.7^{*}$ & $3.9 \pm 2.2^{*}$ \\
$\mathrm{TC}(\mathrm{mmol} / \mathrm{L})$ & $2.8 \pm 1.3$ & $6.2 \pm 2.3^{*}$ & $6.4 \pm 2.5^{*}$ \\
$\mathrm{ALT}(\mathrm{U} / \mathrm{L})$ & $46.5 \pm 8.6$ & $53.4 \pm 6.7$ & $49.4 \pm 10.9$ \\
$\mathrm{AST}(\mathrm{U} / \mathrm{L})$ & $75.6 \pm 19.7$ & $86.1 \pm 15.4$ & $89.2 \pm 14.8$ \\
$\mathrm{BUN}(\mathrm{mmol} / \mathrm{L})$ & $7.9 \pm 2.7$ & $8.4 \pm 2.1$ & $8.0 \pm 1.8$ \\
Scr $(\mu \mathrm{mmol} / \mathrm{L})$ & $35.3 \pm 4.2$ & $37.8 \pm 4.6$ & $38.1 \pm 4.9$ \\
\hline n=15 per group. Data are expressed as the mean \pm SD. BW, body weight; FPG, \\
fasting plasma glucose; KW/BW, kidney weight to body weight ratio; UMA, \\
urinary microablumin; TG, triglyceride; TC, total cholesterol; ALT, alanine \\
aminotransferase; AST, aspartate aminotransferase; BUN, blood urea nitrogen; and \\
Scr, serum creatinine; ${ }^{*}$ < < 0.05 vs. the NC group, \#P < 0.05 vs. the DKD group. NC: \\
normal control; DKD: diabetic kidney disease; TP: triptolide.
\end{tabular}

\section{Triptolide improved renal pathological changes in vivo}

HE, PAS and Masson staining were conducted to explore whether triptolide ameliorated pathological changes in diabetic kidneys. The images showed that diabetic rats treated with triptolide had a significant decrease in renal tubular hypertrophy and glomerular enlargement compared with the DKD group (Figure 1). The accumulation of glycogen shown by PAS staining and tubulointerstitial fibrosis (collagen fibers, blue) shown by Masson staining were increased in 


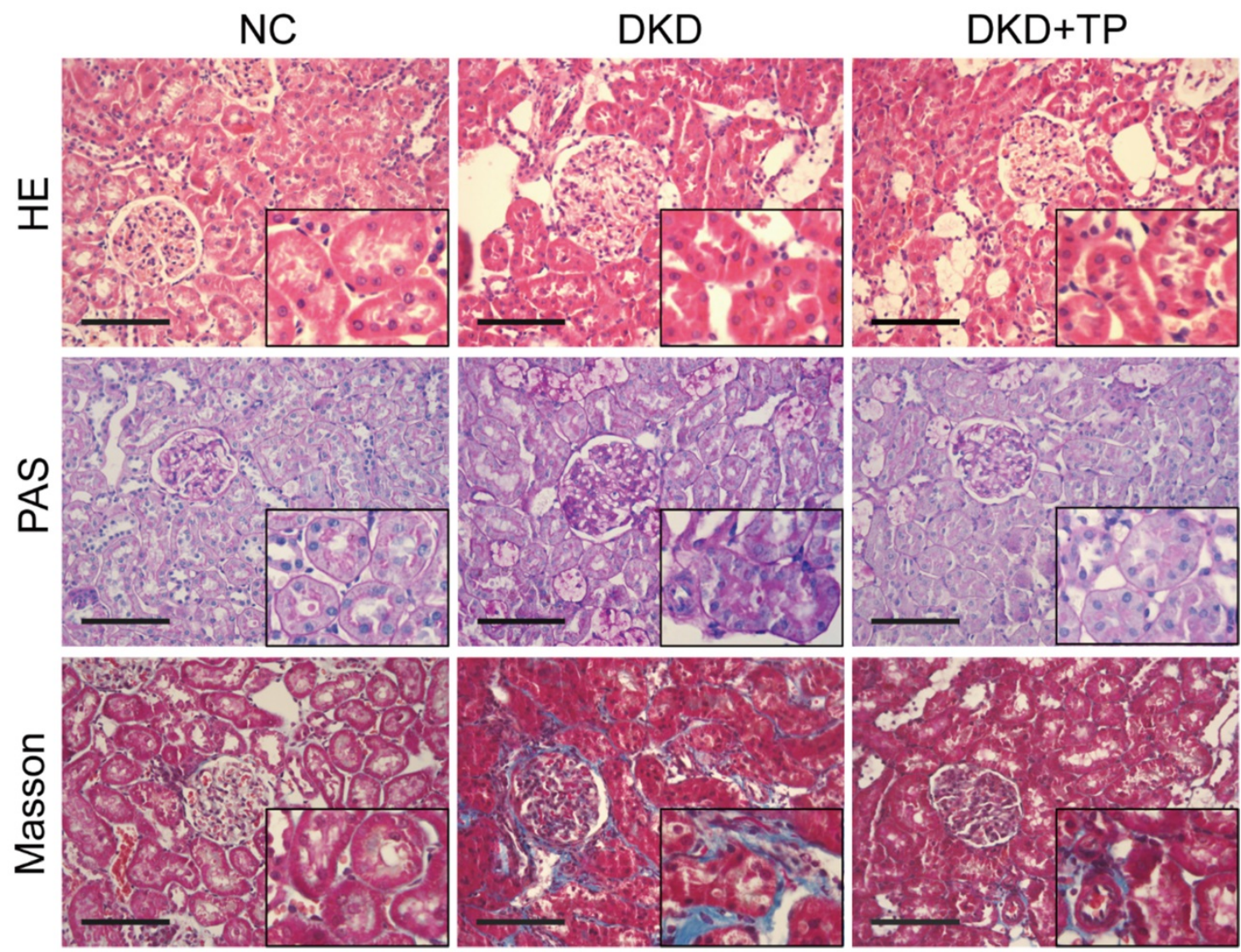

Figure 1. Renal pathological changes in animal subjects. Representative images of hematoxylin and eosin (HE), periodic acid-Schiff (PAS) and Masson's trichrome (Masson) stained kidney sections (inset images indicate augmentative renal tubules). Original magnification is $\times 400$. The scale bar represents $100 \mu \mathrm{m}$. NC: normal control; DKD: diabetic kidney disease; TP: triptolide.

DKD animals relative to the NC group, whereas the signs of glomerular hypertrophy and tubulointerstitial fibrosis were significantly alleviated after a 12-week treatment with triptolide (Figure 1).

\section{Triptolide attenuated renal EMT and regulated the PI3K/AKT signaling pathway in diabetic rats}

We next detected the expression levels of markers related to EMT, such as E-cadherin, vimentin and a-SMA. Immunohistochemistry revealed that the vimentin and a-SMA expression levels were markedly upregulated, but E-cadherin was downregulated in the DKD group compared with the NC group. Triptolide treatment partially inhibited the vimentin and a-SMA expression levels and recovered E-cadherin expression in diabetic rats (Figure 2A). Correspondingly, the vimentin, a-SMA and E-cadherin protein levels displayed similar changes, as detected by Western blot analysis (Figures 2B and C). To further investigate the potential molecular mechanisms regarding the anti-EMT effects of triptolide in vivo, the levels of markers in the PI3K/AKT signaling pathway were examined. As shown in the figure, the PI3K expression levels and ratio of $\mathrm{p}$-AKT to $\mathrm{t}$-AKT (p-AKT/ t-AKT) were obviously increased compared with the NC group, whereas the PTEN levels were decreased in the DKD group. When diabetic animals were treated with triptolide, the PI3K and p-AKT/ t-AKT protein levels were lower and the PTEN levels were higher than in animals without treatment (Figures 2D and E).

\section{Triptolide reduced HG-induced EMT via the PI3K/AKT signaling pathway in vitro}

According to the results measured by the CCK 8 kit, low concentrations of triptolide had no marked influence on the viability of HK-2 cells compared with the control group. However, if the concentrations were greater than $7.5 \mathrm{ng} / \mathrm{mL}$, triptolide significantly impaired cell survival. Therefore, $5 \mathrm{ng} / \mathrm{mL}$ triptolide was chosen to be the appropriate intervention concentration in HK-2 cells (Figure S1A). We first observed that NG-treated cells displayed a cobblestone-like shape, while HG-treated cells transformed into a long spindle-like shape. Interestingly, triptolide-treated cells regained the appearance of epithelial cells and significant morphological changes were not observed between the MA and NG group (Figure S1B). As illustrated 
using immunofluorescence microscopy, HG resulted in evident increases of the vimentin and a-SMA levels and a decrease of E-cadherin expression. In cells treated with triptolide, EMT was greatly improved (Figure 3A). Convincingly, qPCR and Western blot analyses also showed that triptolide relieved the HG-induced upregulation of the vimentin and a-SMA mRNA and protein levels and downregulation of the E-cadherin mRNA and protein levels, respectively (Figures 3B-D). The PI3K and p-AKT/ t-AKT protein levels were relatively increased and PTEN was decreased in HK-2 cells incubated with HG compared with control cells, and triptolide significantly reduced the PI3K and $\mathrm{p}-\mathrm{AKT} / \mathrm{t}-\mathrm{AKT}$ protein levels and increased the PTEN levels (Figures 3E and F). In all cases, the mannitol control showed no apparent differences in HK-2 cells relative to the NG group. These data further confirmed that triptolide also alleviated HG-induced EMT via the PI3K/AKT signaling pathway in HK-2 cells.

\section{Triptolide reduced the expression of miR-188-5p induced by HG and miR-188-5p directly targeted PTEN}

The results obtained from miRNA microarray analysis in human renal mesangial cells demonstrated

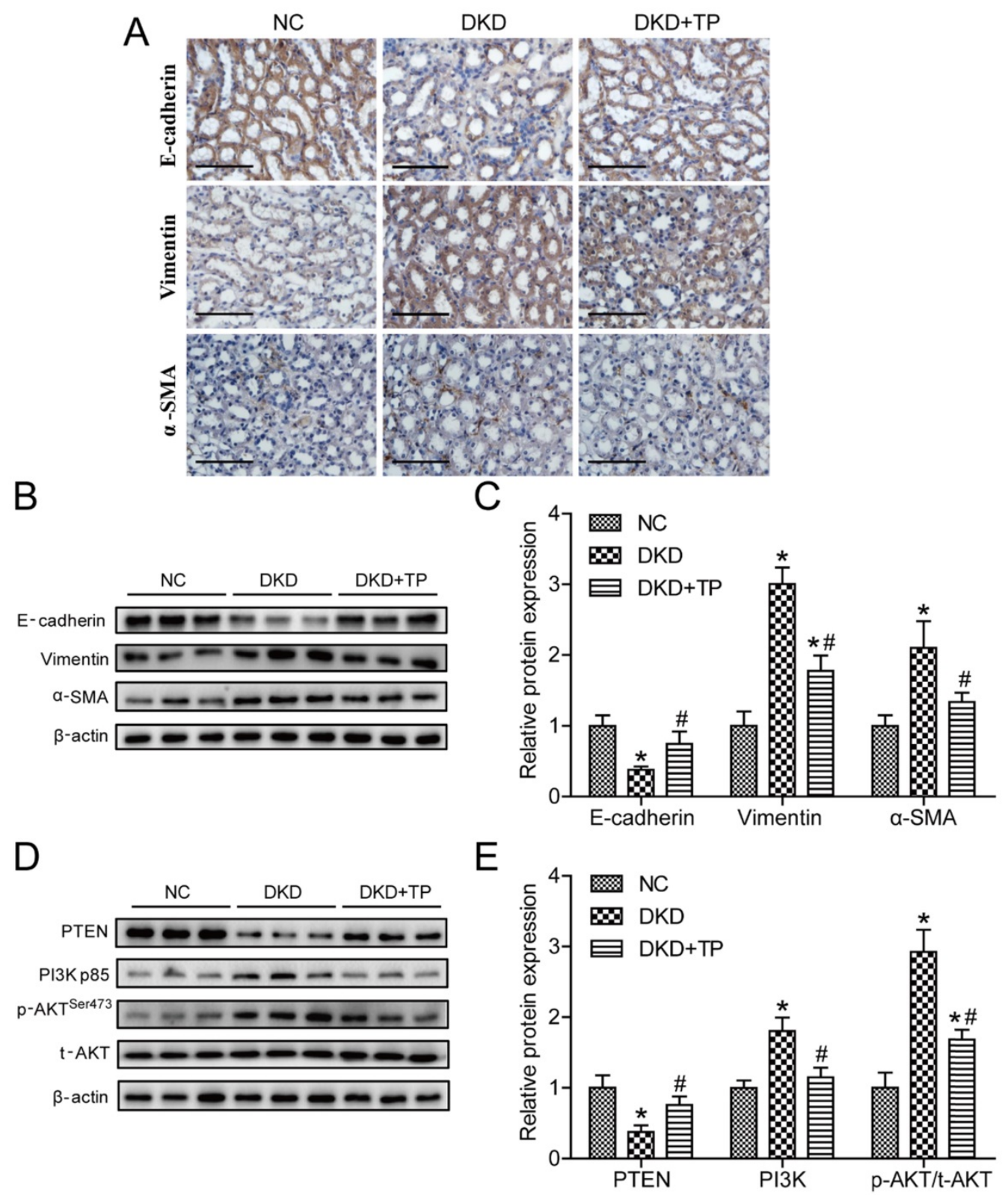

Figure 2. Triptolide reduced renal EMT and inactivated the PI3K/AKT signaling pathway in vivo. (A) Representative images of E-cadherin, vimentin and $\alpha$-SMA by immunohistochemistry from renal tubules. Original magnification is $\times 400$. The scale bar represents $50 \mu m$. (B) Representative E-cadherin, vimentin and $\alpha$-SMA bands by Western blot in rat kidneys. (C) Densitometric analysis of E-cadherin, vimentin and a-SMA by Western blot $(n=5)$. (D) Representative PTEN, PI3K, $p-A K T$ and $t-A K T$ bands by Western blot in rat kidneys. (E) Densitometric analysis of PTEN, PI3K, $\mathrm{P}-\mathrm{AKT}$ and $\mathrm{t}-\mathrm{AKT}$ by Western Blot $(\mathrm{n}=5)$. Data are expressed as the mean \pm SD. $* P<0.05$ vs. the NC group. \#P $<0.05$ vs. the DKD group. NC: normal control; DKD: diabetic kidney disease; TP: triptolide. 
a significant increase in miR-188-5p expression under HG conditions, while triptolide reduced the levels of miR-188-5p compared with cells without intervention (Figure S1C). Subsequently, expression of miR-188-5p was analyzed by qPCR in HK-2 cells. Consistently, the results showed that miR-188-5p expression was apparently elevated in the HG group compared with the NG group and that miR-188-5p expression was decreased after cells were treated with $5 \mathrm{ng} / \mathrm{mL}$ triptolide under HG conditions (Figure 4A). We further detected expression of miR-188-5p in vivo. The miR-188-5p levels in diabetic kidneys were considerably increased, and triptolide significantly decreased this expression (Figure 4B). Next, we attempted to identify the potential target genes of miR-188-5p with TargetScan. We found that PTEN was a predicted target of miR-188-5p because of their complementary sequences (Figure 4C). Dual-luciferase reporter assays were performed to verify whether PTEN expression was hindered by the direct interaction between the PTEN 3'-UTR and miR-188-5p. We observed that ectopic expression of miR-188-5p significantly repressed the luciferase activity of the wild-type PTEN-3'-UTR, but had no effect on the luciferase activity of the mutant PTEN-3'-UTR. These results confirmed that PTEN was a direct target of miR-188-5p (Figure 4D). Meanwhile, the qPCR and Western blot results demonstrated that the PTEN mRNA and protein levels were significantly decreased by HG incubation and that triptolide enhanced the PTEN mRNA and protein levels (Figures 2D, 2E and 4E).

B
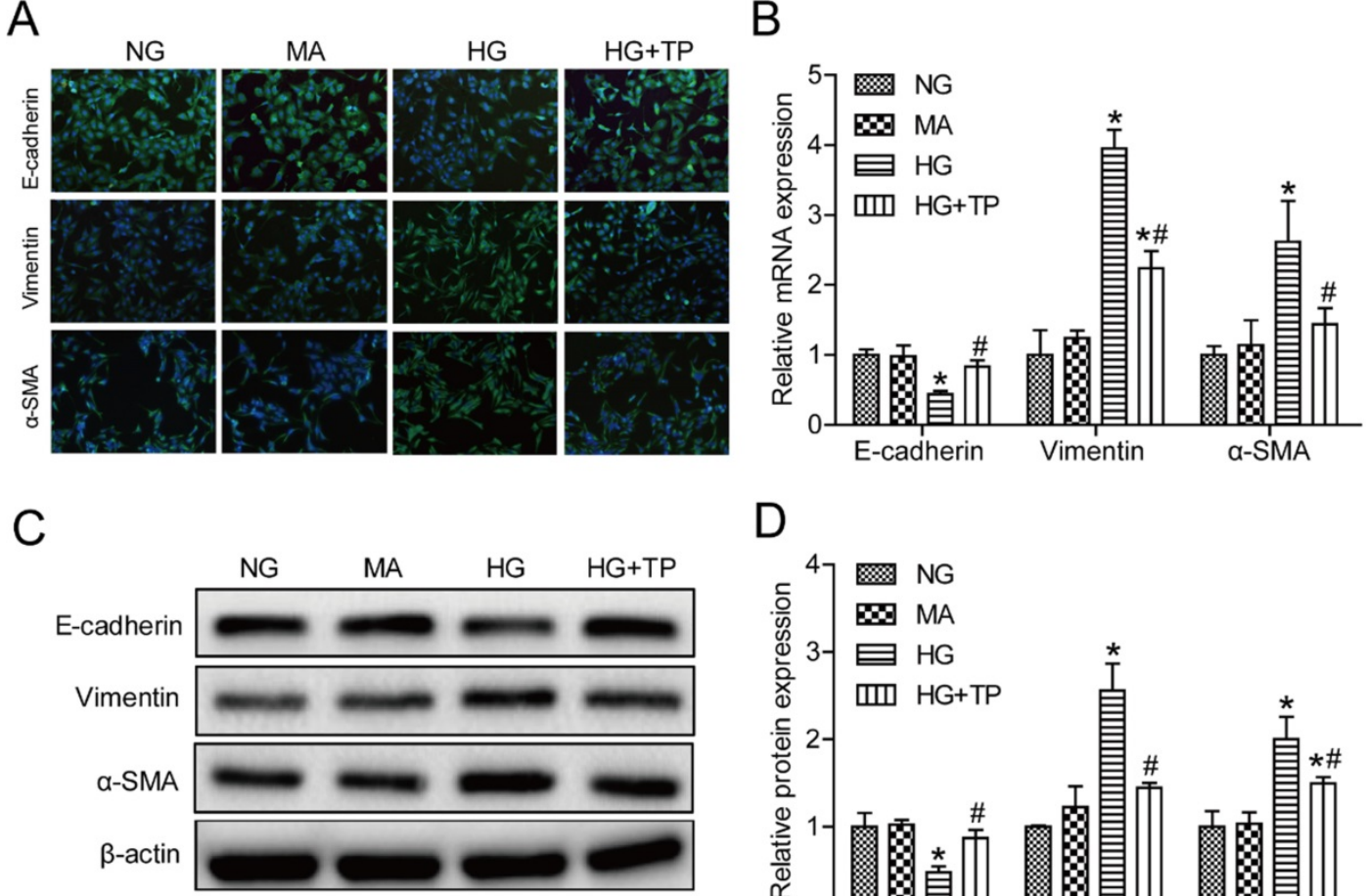

$\mathrm{E}$

$\mathrm{D}$

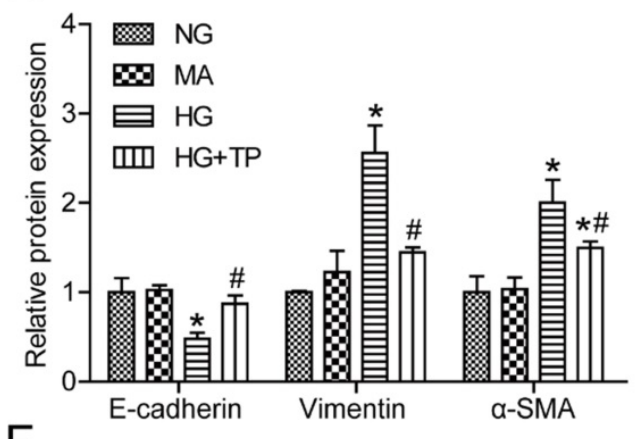

$\mathrm{F}$

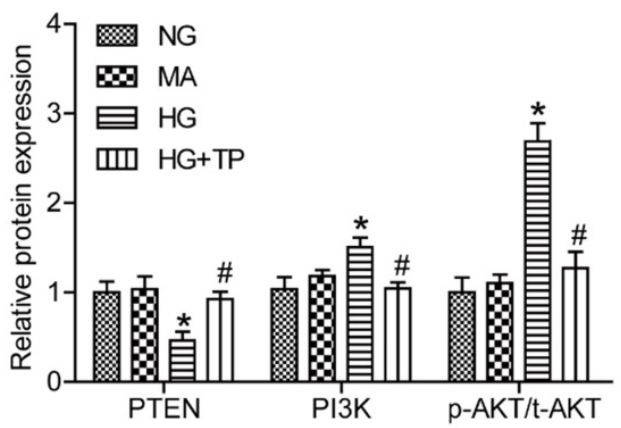

Figure 3. Triptolide modulated the expression of E-cadherin, vimentin and $\alpha$-SMA by inactivating the PI3K/AKT signaling pathway in HK-2 cells. (A) Representative images of E-cadherin (green), vimentin (green) and a-SMA (green) by immunofluorescence in HK-2 cells. Blue staining refers to the DAPI-stained nuclei. Original magnification is $\times 200$. (B) Quantification of $E$-cadherin, vimentin and $\alpha$-SMA gene expression in HK-2 cells $(n=4)$. (C) Representative $E$-cadherin, vimentin and $\alpha$-SMA bands by Western blot in HK-2 cells. (D) Densitometric analysis of E-cadherin, vimentin and $\alpha$-SMA by Western blot ( $n=4)$. (E) Representative PTEN, PI3K, $P$-AKT and t-AKT bands by Western blot in HK-2 cells. (F) Densitometric analysis of PTEN, PI3K, p-AKT and t-AKT by Western Blot ( $=4)$. Data are expressed as the mean \pm SD. *P < 0.05 vs. the NG group. \#P< 0.05 vs. the HG group. NG: normal glucose; MA: mannitol; HG: high glucose; TP: triptolide. 
A

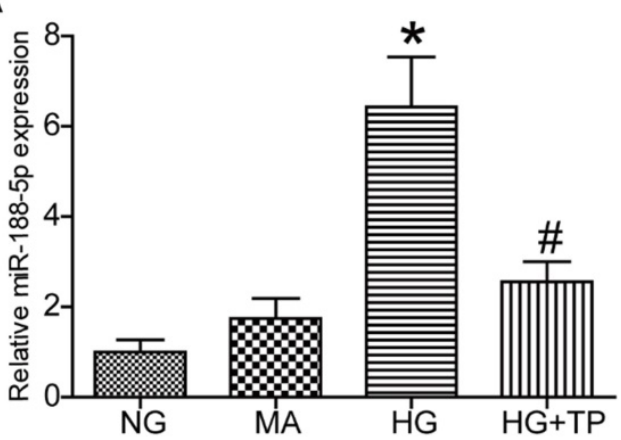

$\mathrm{B}$

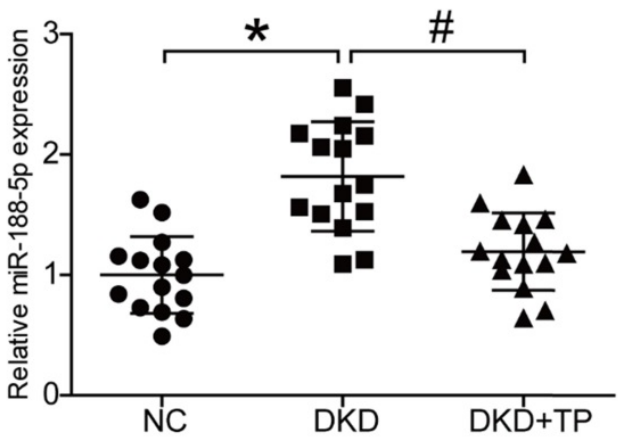

C
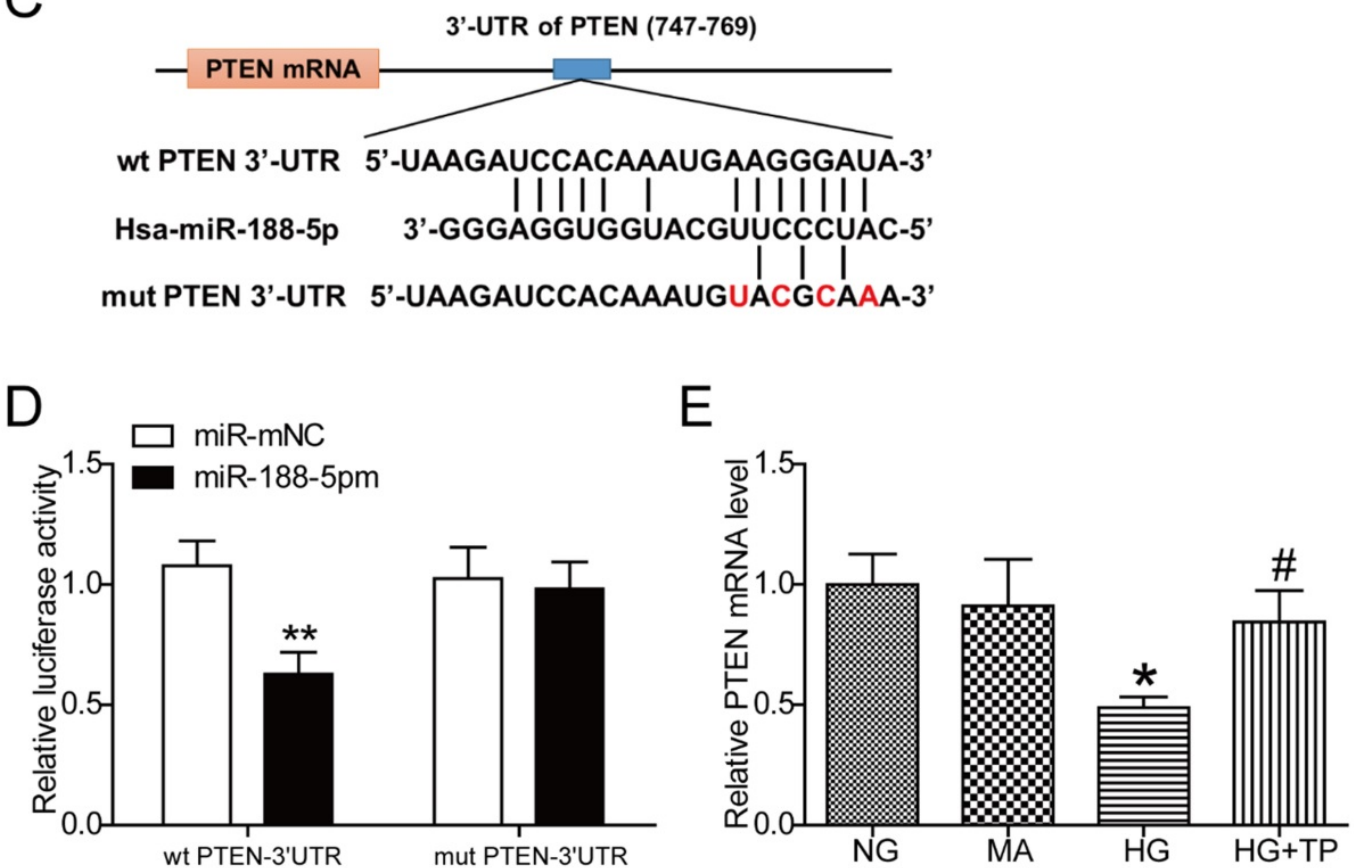

Figure 4. PTEN is a direct target of miR-188-5p and triptolide can promote PTEN expression. (A) Quantification of miR-188-5p gene expression in the HK-2 cells $(n=4)$. (B) Quantification of miR-188-5p gene expression in rat kidneys $(n=15)$. (C) miR-188-5p sequence and its potential binding sites in the wild-type PTEN-3'-UTR. The complementary binding site was replaced in the mutant PTEN 3'-UTR. (D) Dual-luciferase reporter assay with wild-type PTEN-3'-UTR and mutated PTEN- 3'-UTR reporter gene in 293T cells transfected with miR-188-5p mimic or miR-mNC for $24 \mathrm{~h}(\mathrm{n}=4)$. (E) Quantification of PTEN gene expression in the HK-2 cells ( $\mathrm{n}=4)$. Data are expressed as the mean \pm SD. ${ }^{* * P}<0.01$ vs. the miR-mNC group. ${ }^{*} P<0.05$ vs. the NG group. \# $P<0.05$ vs. the HG group. NC: normal control; DKD: diabetic kidney disease; TP: triptolide; NG: normal glucose; MA: mannitol; HG: high glucose; miR-188-5pm: miR-188-5p mimic; miR-mNC: negative control of miR-188-5p mimic.

\section{miR-188-5p inhibitor alleviated HG-induced EMT in HK-2 cells}

To further elucidate whether miR-188-5p is involved in mediating HG-induced EMT by targeting PTEN and affecting the activity of the PI3K/AKT signaling pathway, we constructed a miR-188-5p knockout model in HK-2 cells. When cells were transfected with a miR-188-5p inhibitor prior to HG conditions, miR-188-5p expression was reduced by almost $60 \%$ compared with cells transfected with the miR-188-5p inhibitor negative control (Figure S1D). PTEN protein expression was also increased and the $\mathrm{PI} 3 \mathrm{~K}$ and $\mathrm{p}-\mathrm{AKT} / \mathrm{t}-\mathrm{AKT}$ levels were decreased after deletion of miR-188-5p in HK-2 cells (Figures 5A and B). Moreover, we found that abrogation of miR-188-5p expression caused a considerable increase in the
E-cadherin protein expression levels, but a decrease in the vimentin and a-SMA levels (Figures $5 \mathrm{C}$ and D). These data suggest that the miR-188-5p inhibitor had the same effect as triptolide; thus, miR-188-5p is indispensable for regulation of HG-induced EMT.

\section{miR-188-5p mimic reversed the anti-EMT effect of triptolide in HK-2 cells}

According to the results mentioned above, we speculated that the miR-188-5p mimic might antagonize the effects of triptolide. Thus, we next employed a miR-188-5p mimic to overexpress miR-188-5p in HK-2 cells. As expected, themiR-188-5p levels in cells transfected with the miR-188-5p mimics was approximately 45 times higher than in cells transfected with the miR-188-5p mimic negative 
control (Figure S1E). Meanwhile, Western blot analysis demonstrated that miR-188-5p enrichment reduced PTEN expression but increased the PI3K and p-AKT/t-AKT levels in HK-2 cells (Figures 6A and B). Furthermore, miR-188-5p overexpression decreased the E-cadherin protein expression levels, but
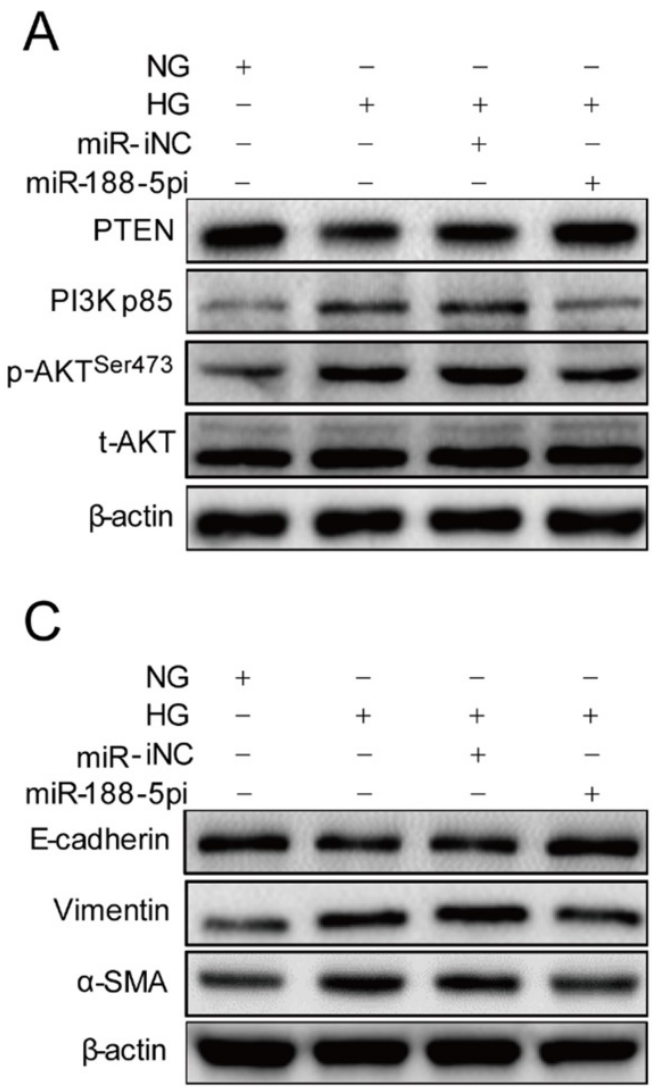

increased the vimentin and a-SMA levels (Figures 6C and D). These results indicated that the miR-188-5p mimics partially offset the anti-EMT effects of triptolide by activating the PI3K/AKT signaling pathway.

B

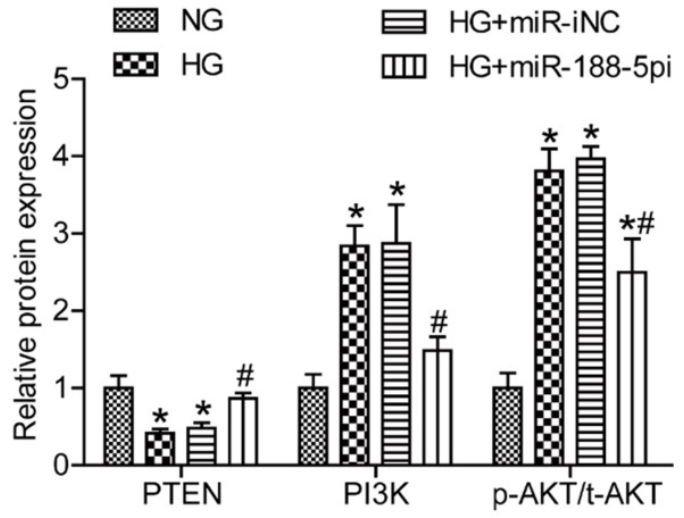

$\mathrm{D}$

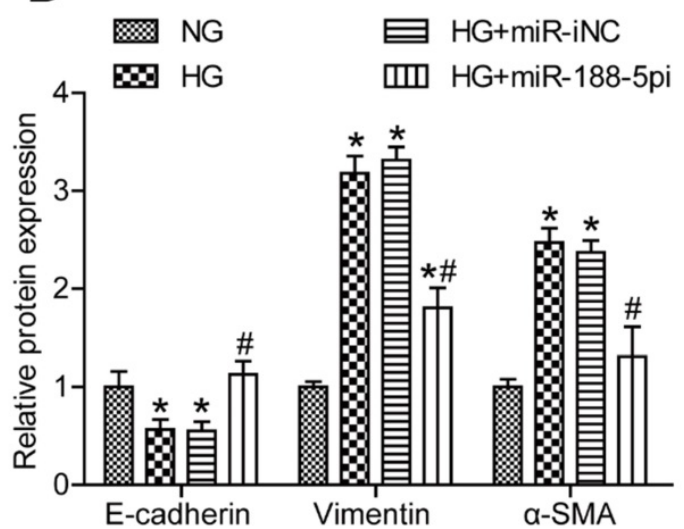

Figure 5. miR-1 88-5p downregulation alleviated HG-induced EMT in vitro. (A) Representative PTEN, PI3K, $\mathrm{p}-\mathrm{AKT}$ and $t-A K T$ bands by Western blot in HK-2 cells. (B) Densitometric analysis of PTEN, PI3K, p-AKT and t-AKT by Western blot $(n=4)$. (C) Representative E-cadherin, vimentin and $\alpha-S M A$ bands by Western blot in HK-2 cells. (D) Densitometric analysis of $E$-cadherin, vimentin and $\alpha$-SMA by Western blot $(n=4)$. Data are expressed as the mean $\pm S D$. $* P<0.05$ vs. the NG group. $\# P<0.05$ vs. the HG group. NG: normal glucose; MA: mannitol; HG: high glucose; TP: triptolide; miR-188-5pi: miR-188-5p inhibitor; miR-iNC: negative control of miR-188-5p inhibitor.

Table 2. Primers used for transcript quantification by qPCR.

\begin{tabular}{ll}
\hline Gene $^{\mathrm{a}}$ & Primer sequences $\left(5^{\prime} \text { - }^{\prime}\right)^{\mathrm{b}}$ \\
\hline Hs/Rs miR-188-5p Stemloop & GTCGTATCCAGTGCAGGGTCCGAGGTATTCGCACTGGATACGACCCCTCC \\
Hs/Rs miR-188-5p F & GGTCCCATCCCTTGCATGGTG \\
Hs/Rs RNU6 Stemloop & GTCGTATCCAGTGCAGGGTCCGAGG \\
Hs/Rs RNU6 F & TGCGGGTGCTCGCTTCGGCAGC \\
Universal primer R & CCAGTGCAGGGTCCGAGGT \\
Hs E-cadherin F & CCACCAGATGACGATACCCG \\
Hs E-cadherin R & GCTTCAGAACCACTCCCCTC \\
Hs Vimentin F & GGGACCTCTACGAGGAGGAG \\
Hs Vimentin R & CGCATTGTCAACATCCTGTC \\
Hs a-SMA F & ACTGCCTTGGTGTGTGACAA \\
Hs a-SMA R & TCCCAGTTGGTGATGATGCC \\
Hs PTEN F & ACTCTTTATGCGCTGCGG \\
Hs PTEN R & ACAGCGGCTCAACTCTCAAA \\
Hs GAPDH F & CTCCTCCACCTTTGACGCTG \\
Hs GAPDH R & TCCTCTTGTGCTCTTGCTGG \\
\hline
\end{tabular}

a Hs, human; Rs, rat.

${ }^{b} \mathrm{~F}$, forward primer; $\mathrm{R}$, reverse primer. 

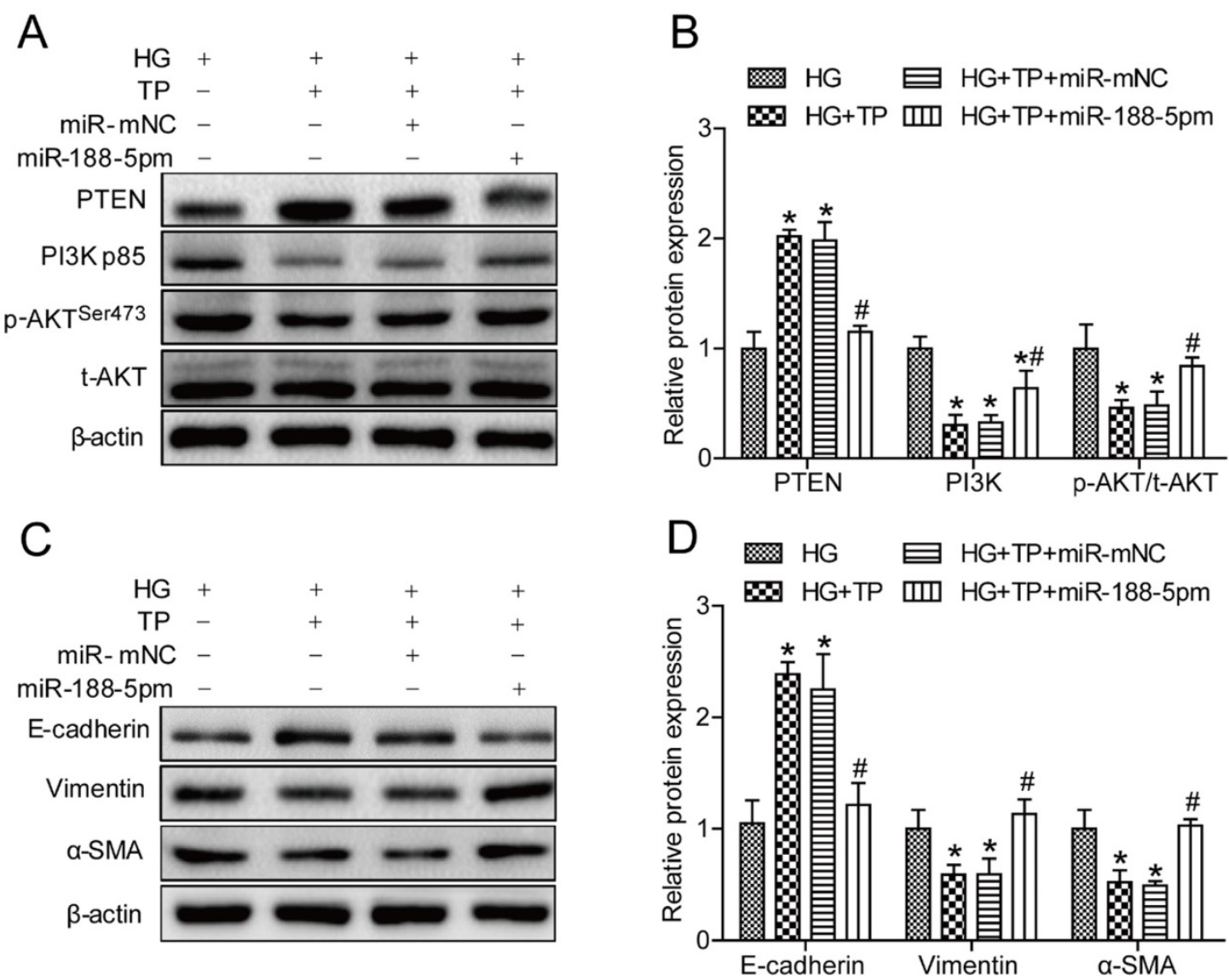

Figure 6. miR-188-5p overexpression reversed the anti-EMT effects of triptolide in vitro. (A) Representative PTEN, PI3K, $\mathrm{P}-\mathrm{AKT}$ and $\mathrm{t}-\mathrm{AKT}$ bands by Western blot in HK-2 cells. (B) Densitometric analysis of PTEN, PI3K, p-AKT and t-AKT by Western blot $(n=4)$. (C) Representative E-cadherin, vimentin and $\alpha$-SMA bands by Western blot in HK-2 cells. (D) Densitometric analysis of E-cadherin, vimentin and $\alpha-S M A$ bands by Western blot $(n=4)$. Data are expressed as the mean $\pm S D$. $* P<0.05$ vs. the HG group. \#P $<0.05$ vs. the HG+TP group. NG: normal glucose; MA: mannitol; HG: high glucose; TP: triptolide; miR-188-5pm: miR-188-5p mimic; miR-mNC: negative control of miR-188-5p mimic.

\section{Discussion}

Our study was designed to investigate the mechanistic actions of triptolide towards renal EMT in DKD. Mesangial expansion, thickening of the glomerular basement membrane, and glomerular and tubulointerstitial fibrosis are characteristic structural changes in DKD [26, 27]. Previous studies have demonstrated that triptolide, the major active ingredient in $\mathrm{TwHF}$, has a therapeutic effect on rats with DKD [23, 24]. It has been confirmed that triptolide attenuates renal interstitial fibrosis in rats with unilateral ureteral obstruction [28]. In the present study, triptolide remarkably decreased albuminuria and beneficially altered the renal histopathological changes observed in a DKD model, which are consistent with previous studies [24, 29].

It is well known that EMT is closely associated with the progress of renal fibrosis in DKD [30]. In our study, morphological changes in HK-2 cells under HG conditions were observed first. Using histochemical and biochemical approaches, EMT was induced by hyperglycemia or HG in DKD rats and HK-2 cells. Liu et al. provided experimental evidence of effective inhibition of hypoxia-induced EMT by triptolide in pancreatic cancer [31]. Similarly, we proved that triptolide could alleviate renal EMT both in vivo and in vitro. So far, many studies have revealed that the PI3K/AKT signaling pathway plays a crucial role in EMT during DKD. LY294002, a PI3K inhibitor, prevented HG-induced EMT in NRK-52E cells by suppressing AKT phosphorylation $[32,33]$. However, it was unclear whether the PI3K/AKT signaling pathway was involved in the anti-EMT effects of triptolide in DKD rats and HK-2 cells. Our findings showed that triptolide notably increased the inactivation of the PI3K/AKT pathway in vivo and in vitro. Therefore, we discovered that the PI3K/AKT pathway was the potential mechanism to mitigate the effects by triptolide on EMT.

MiRNAs play a crucial part in numerous diseases, including diabetes, cardiovascular diseases, kidney diseases and cancers[34]. Recently, a large number of studies have revealed that aberrant miRNA expression in the kidney may result in the development and progression of DKD [35]. For example, Zhao et al. found that miR-23b expression 
was decreased in vitro and in vivo and that G3BP2 was a downstream target mRNA of miR-23b, which regulate pathogenic processes in DKD [36]. Additionally, several miRNAs are considered to be important mediators of renal EMT, such as miR-21, Let-7d, miR-141 and miR-590 [10, 37-39]. Moreover, miRNAs also serve as regulatory elements regarding the effects of drug interventions in DKD [40,41]. Here, we detected miRNA expression using a miRNA microarray technology and subsequently quantified the expression of interesting miRNAs using qPCR. The results showed that the increase in miR-188-5p expression in HK-2 cells exposed to HG was significantly reversed by triptolide, which was consistent with the miR-188-5p levels in rat kidneys. Although other miRNAs also showed differential expression (Figure S1C), few studies reported that whether miR-188-5p was involved in regulating tubular EMT in DKD through PI3K/AKT pathway. Thence miR-188-5p was selected as a candidate miRNA in this study.

As is widely known, miRNAs exert their biological effects by binding to the complementary 3 '-UTR sequences of target mRNAs at the posttranscriptional level [42]. Next, we attempted to find the possible target gene of miR-188-5p. Above results have proved that triptolide reduced glucose-stimulated PI3K/AKT pathway activation and miR-188-5p expression in HK-2 cells. We speculated that miR-188-5p has an effect on one of signal molecules in PI3K/AKT pathway. According to bioinformatics analyses using TargetScan, PTEN was deemed to be a target miR-188-5p candidate. The results from the dual-luciferase reporter assay also confirmed this prediction. PTEN is a tumor suppressor that mainly blocks the PI3K/AKT pathway in a negative-regulation manner and is usually inactivated in somatic cancers [43]. Moreover, PTEN has been found to exert a crucial role in EMT. For example, PTEN is a direct target of miR-130b and participates in EMT-induced metastasis in hepatocellular carcinoma [17]. Decreased PTEN expression regulated by miR-494 also promotes cyclosporine-induced EMT in HK-2 cells and kidneys from C57BL/6J mice, and $\mathrm{BpV}$, a PTEN inhibitor, accelerates EMT in renal tubular cells $[15,18]$. As a miR-188-5p target gene, it is easy to understand that changes in PTEN expression occur in contrast to miR-188-5p expression. Nevertheless, whether triptolide ameliorates renal EMT via a miR-188-5p-associated PI3K/AKT pathway remained unknown. In the current in vitro study, it was shown that miR-188-5p ablation preserved HK-2 cells against PI3K/AKT pathway activation and HG-induced EMT. Second, we discovered that miR-188-5p enrichment weakened the therapeutic action of triptolide during HG-related EMT. Therefore, these results demonstrated that miR-188-5p was key for governing the effects of triptolide on renal EMT through the PI3K/AKT pathway.

In summary, our data suggest that triptolide may be an effective therapeutic approach in DKD. The increase in PTEN levels and decrease in PI3K/AKT pathway activation mediated by miR-188-5p inhibition is a potential mechanism by which triptolide could prevent renal EMT in DKD. Thereby, targeting miR-188-5p might be a novel therapy for triptolide in renal tubulointerstitial fibrosis.

However, some limitations were present in this study. Even though we demonstrated that triptolide improved renal structure and renal tubular EMT, we did not develop any miR-188-5p overexpression or suppression models. Further studies may solve this problem by using miR-188-5p-inhibitor/mimictreated or gene-knockout animal models. DKD rats also showed glomerular fibrosis, and we did not assess the expression of miR-188-5p in mesangial cells and podocytes. It is unclear whether abnormal miR-188-5p expression is connected with glomerular injury. Furthermore, one miRNA targets numerous mRNAs and one mRNA is regulated by various miRNAs. This complicated network means that other miRNAs, target molecules and pathways may participate in the triptolide mechanism modulating EMT. Thus, more studies are urgently required to address the above problems.

\section{Materials and Methods}

\section{Animals}

Six-week-old Male Sprague-Dawley rats weighing $160 \pm 8 \mathrm{~g}$ were purchased from Beijing HFK Bioscience Co., Ltd. (Beijing, China). This study was performed in accordance with the recommendations from the Guide for the Care and Use of Laboratory Animals of the National Institutes of Health and the Animal Welfare Act guidelines. The protocol was approved by the ethical committee of Tianjin Medical University (Tianjin, China). All rats were maintained under pathogen-free conditions $(12 \mathrm{~h}$ light/dark cycles, $55 \% \pm 10 \%$ humidity, and $22^{\circ} \mathrm{C} \pm 2{ }^{\circ} \mathrm{C}$ temperature). Fifteen rats were randomly chosen to receive a standard diet (NC group, $n=15$ ), while others were fed a high-fat diet (HFD group, $n=38$ ) for 8 weeks. The HFD consisted of $78.7 \%$ standard diet, $10 \%$ glucose, $10 \%$ animal fat, $1 \%$ total cholesterol and $0.3 \%$ sodium cholate. Diabetes was induced in the HFD group by a tail intravenous injection with a single dose of streptozocin dissolved in sodium citrate buffer (30 mg/kg, $\mathrm{pH} 4.5)$, while the NC group was 
administered isometric sodium citrate buffer. When the random blood glucose was $\geq 16.7 \mathrm{mmol} / \mathrm{L}$ after the streptozocin injection, the diabetic model was considered to be successful. Thirty rats that met the diabetes criteria were randomly assigned to the DKD $(n=15)$ and DKD +TP $(n=15)$ groups. DKD +TP rats were gavaged with triptolide at a dose of 200 $\mu \mathrm{g} / \mathrm{kg} / \mathrm{d}$ for 12 weeks, while the NC and DKD groups received equal amounts of saline.

\section{Cell culture and treatment}

Human proximal tubular epithelial cells (HK-2 cells, American Type Culture Collection, USA) were maintained in DMEM medium (Hyclone, USA) containing $10 \%$ fetal bovine serum (Gibco, USA), 200 $\mathrm{U} / \mathrm{mL}$ penicillin, and $200 \mu \mathrm{g} / \mathrm{mL}$ streptomycin (Solarbio, China) in an incubator with a humidified atmosphere of $5 \% \mathrm{CO}_{2}$ at $37^{\circ} \mathrm{C}$. The cells were divided into the following groups, which contained various additives in their media: (1) the NG group was incubated in DMEM containing $5.5 \mathrm{mmol} / \mathrm{L}$ glucose, (2) the MA group was incubated in DMEM containing $5.5 \mathrm{mmol} / \mathrm{L}$ glucose and $24.5 \mathrm{mmol} / \mathrm{L}$ mannitol, (3) the HG group was incubated in DMEM containing 30 mmol/L glucose, and (4) the HG+TP group was incubated with $5 \mathrm{ng} / \mathrm{mL}$ triptolide in DMEM containing $30 \mathrm{mmol} / \mathrm{L}$ glucose. Each group was incubated under different conditions for $48 \mathrm{~h}$ as previously reported $[15,44]$.

\section{Biochemical analysis}

At the end of the treatment, $24 \mathrm{~h}$ urine samples from all rats were obtained for the measurement of urinary microablumin (UMA). All overnight-fasted animals were weighed and then anesthetized with an intraperitoneal injection of chloral hydrate. Blood samples were collected from the retroorbital venous plexus for biochemical analyses. Blood glucose, UMA, total triglyceride (TG), total cholesterol (TC), alanine transaminase (ALT), aspartate transaminase (AST), blood urea nitrogen (BUN) and serum creatinine (Scr) were assessed by an automatic biochemistry analyzer (Roche, Germany). The kidneys were immediately collected, weighed, and fixed in 10\% neutral formalin or frozen in liquid nitrogen and then stored at $-80^{\circ} \mathrm{C}$ until further experiments.

\section{Histological analysis}

Five micrometer thick kidney sections embedded in paraffin were cut for histological analysis. The sections were stained with hematoxylin and eosin (HE), periodic acid-Schiff (PAS) and Masson's trichrome (Masson) staining kits (Leagene Biotechnology, China) according to the manufacturer's recommendations.

\section{Immunohistochemistry staining of renal tissues}

Formalin-fixed and paraffin-embedded kidney slides were deparaffinized with xylene, hydrated with graded ethanol, subjected to antigen retrieval at $95^{\circ} \mathrm{C}$, blocked with $3 \% \mathrm{H}_{2} \mathrm{O}_{2}$ and incubated overnight at $4^{\circ} \mathrm{C}$ with the following primary antibodies: anti-E-cadherin (1: 100, no.3195, Cell Signaling Technology, USA), anti-vimentin (1: 100, no.10366-1-AP, Proteintech, China), and anti-a-SMA (1: 100, no. 7817, Abcam, USA). Subsequently, the sections were incubated with the appropriate secondary antibody, visualized with a DAB kit and counterstained with hematoxylin.

\section{Western blot analysis}

Proteins from kidney tissues and HK-2 cells were extracted using RIPA buffer (Solarbio, China) following the manufacturer's instructions, and the protein concentrations were determined using a BCA protein assay kit (Solarbio, China). Proteins from each experimental group were separated by SDS-PAGE gels, transferred onto PVDF membranes, blocked with $5 \%$ defatted milk for $2 \mathrm{~h}$ at room temperature and incubated overnight at $4^{\circ} \mathrm{C}$ with the following primary antibodies: anti-E-cadherin (1:1000, no. 3195, Cell Signaling Technology, USA), anti-vimentin (1:2000, no. 10366-1-AP, Proteintech, China), anti-a-SMA (1:200, no. 7817, Abcam, USA), anti-PTEN (1:1000, no. 9559, Cell Signaling Technology, USA), anti-PI3K p85 (1:1000, no. 4257, Cell Signaling Technology, USA), anti-p-AKT Ser473 (1:1000, no.4060, Cell Signaling Technology, USA), anti-t-AKT (1:1000, no. 4691, Cell Signaling Technology, USA), and anti- $\beta$-actin (1:5000, no. KM9001, Sungene Biotech, China). After washing, the HRP-conjugated secondary anti-rabbit/mouse antibody (Sungene Biotech, China) was used at a 1:3000 dilution for $1 \mathrm{~h}$ at room temperature. Protein bands were visualized with an ECL kit (Advansta, USA). Densitometry analyses of the bands were quantified with Image J software. The housekeeping protein $\beta$-actin was used as a loading control.

\section{CCK 8 assay}

The cell counting kit 8 (CCK 8) was used to obtain the optimal drug concentration for the therapeutic effect instead of impairing cells' growth. Cells were plated in 96-well plates at the density of $5 \times 10^{4}$ / well. Different concentrations of triptolide $(0$, $0.5,1,2.5,5,7.5,10,20$ and $50 \mathrm{ng} / \mathrm{mL}$ ) were added to the medium for $48 \mathrm{~h}$. Next, cells were incubated with $10 \mu \mathrm{L}$ of CCK 8 reagent for 2 hours, and the absorbance at $450 \mathrm{~nm}$ was measured. Assays were 
performed with 6 replicate wells for each group, and the experiment was conducted in triplicate.

\section{Immunofluorescence staining of HK-2 cells}

Cells were plated on plastic cover slips for immunocytochemistry. After incubation, cells were fixed with $4 \%$ paraformaldehyde for $10 \mathrm{~min}$ and permeabilized with $0.1 \%$ Triton X-100 for $30 \mathrm{~min}$. Subsequently, cells were blocked with 5\% BSA for 30 min and incubated with the following primary antibodies overnight at $4^{\circ} \mathrm{C}$ : anti-E-cadherin (1:50, no. 3195, Cell Signaling Technology, USA), anti-vimentin (1:50, no. 10366-1-AP, Proteintech, China), and anti-a-SMA (1:50, no. 7817, Abcam, USA). Next, cells were incubated with the corresponding FITC-conjugated secondary antibodies for $30 \mathrm{~min}$ and DAPI for $2 \mathrm{~min}$ in the dark. Finally, images were captured using a fluorescence microscope equipped with a digital camera.

\section{RNA isolation and Quantitative PCR}

Total RNA was extracted from HK-2 cells and kidney samples using the E.Z.N.A.TM HP total RNA Kit (Omega, USA) according to the manufacturer's instructions. cDNA was synthesized using a reverse transcription system kit (Thermo, USA). Quantitative PCR (qPCR) was performed with a SYBR Green PCR reagent kit (Sangon Biotech, China) on a CFX96 real-time PCR system (Bio-Rad, USA). Finally, the absorption value of SYBR Green fluorescence in each sample was detected. The miRNA and RNA expression levels were normalized to those of small nuclear RNA (RNU6) and a housekeeping gene $(\mathrm{GAPDH})$, respectively. All of the primers, which were synthesized by AuGCT Biotechnology (Beijing, China), are listed in Table 1.

\section{Luciferase reporter assay}

The predicted 3'-UTR sequence of PTEN interacting with miR-188-5p and mutated sequences within the predicted target sites were synthesized and inserted into the pRL-TK control plasmid containing a Renilla luciferase gene (Promega, USA). 293T cells were seeded into 96-well plates prior to transfection, followed by co-transfection with $100 \mathrm{ng} /$ well pRL-TK plasmid, wild-type PTEN-3'-UTR or mutant PTEN-3'-UTR reporter plasmid and $5 \mathrm{pmol} /$ well miR-mNC or miR-188-5pm for $24 \mathrm{~h}$. The luciferase activities of the cell lysates were measured using a Dual-Luciferase Assay System (Promega, USA). The Renilla luciferase activity of each transfected well was used as an internal control for normalization.

\section{miRNA mimic and inhibitor transfections}

HK-2 cells were transfected with a miR-188-5p inhibitor (miR-188-5pi), miR-188-5p mimic
(miR-188-5pm), or miRNA inhibitor (miR-iNC) and miRNA mimic (miR-mNC) as negative controls (Genepharma, Shanghai, China) at a final concentration of $50 \mathrm{nmol} / \mathrm{L}$ using the transfection reagent Lipofectamine 2000 (Invitrogen, USA) following the manufacturer's protocol. The transfected cells were starved via incubation with serum-free medium overnight prior to incubating the cells with $30 \mathrm{mM}$ glucose or medium containing 30 $\mathrm{mM}$ glucose and $5 \mathrm{ng} / \mathrm{mL}$ triptolide for $48 \mathrm{~h}$.

\section{Statistical analysis}

GraphPad Prism 5.0 software was used for statistical analysis. The values are shown as the mean $\pm \mathrm{SD}$. The significance of the data was analyzed using one-way analysis of variance (ANOVA). $P<0.05$ was considered to indicate statistical significance.

\section{Abbreviations}

ALT: alanine aminotransferase; AKT: protein kinase B; AST: aspartate aminotransferase; BUN: blood urea nitrogen; BW: body weight; CCK 8: cell counting kit 8; DKD: diabetic kidney disease; EMT: epithelial mesenchymal transition; FPG: fasting plasma glucose; HE: hematoxylin and eosin; HFD: high-fat diet; HG: high glucose; HK-2: human kidney proximal tubular epithelial cells; KW/BW: kidney weight to body weight ratio; MA: mannitol; miRNAs: microRNAs; mRNAs: messenger RNAs; NC: normal control; NG: normal glucose; PAS: periodic acid-Schiff; PI3K: phosphatidylinositol 3-kinase; PTEN: phosphatase and tensin homologue deleted chromatosome ten; Scr: serum creatinine; a-SMA: a-Smooth muscle actin; TC: total cholesterol; TG: triglyceride; TP: triptolide; TwHF: Tripterygium wilfordii Hook F; UMA: urinary microablumin; 3'-UTR: 3'-untransliated region.

\section{Supplementary Material}

Supplementary figure.

http://www.ijbs.com/v14p1545s1.pdf

\section{Acknowledgments}

This work was supported by the National Natural Science Foundation of China (no. 81273915 and 81470187), Natural Science Foundation of Tianjin (no. 15ZXHLSY00460 and no. 14JCZDJC33700) and the Science\&Technology Development Fund of Tianjin Education Commission for Higher Education 2017KJ210.

\section{Author Contributions}

Liming Chen and Bei Sun contributed to designing the experiment, interpreting the results and revising the manuscript critically. Mei Xue conducted 
the experiment, analyzed the data and drafted the manuscript. Ying Cheng participated in the discussion of the results and revision of the manuscript. Fei Han, Yunpeng Chang and Yang Yang assisted in analyzing the data. Xiaoyu Li, Li Chen and Yunhong $\mathrm{Lu}$ assisted in carrying out the experiment.

\section{Competing Interests}

The authors have declared that no competing interest exists.

\section{References}

1. Himmelfarb J, Tuttle KR. New therapies for diabetic kidney disease. The New England journal of medicine. 2013; 369: 2549-50.

2. Wang L, Gao P, Zhang M, Huang Z, Zhang D, Deng Q, et al. Prevalence and Ethnic Pattern of Diabetes and Prediabetes in China in 2013. Jama. 2017; 317: 2515-23.

3. Guo K, Zhang L, Zhao F, Lu J, Pan P, Yu H, et al. Prevalence of chronic kidney disease and associated factors in Chinese individuals with type 2 diabetes: Cross-sectional study. Journal of diabetes and its complications. 2016; 30: 803-10.

4. Kim MK. Treatment of diabetic kidney disease: current and future targets. The Korean journal of internal medicine. 2017; 32: 622-30.

5. Su Z, Widomski D, Ma J, Namovic M, Nikkel A, Leys L, et al. Longitudinal Changes in Measured Glomerular Filtration Rate, Renal Fibrosis and Biomarkers in a Rat Model of Type 2 Diabetic Nephropathy. American journal of nephrology. 2016; 44: 339-53.

6. Bhatt K, Lanting LL, Jia Y, Yadav S, Reddy MA, Magilnick N, et al. Anti-Inflammatory Role of MicroRNA-146a in the Pathogenesis of Diabetic Nephropathy. Journal of the American Society of Nephrology : JASN. 2016; 27: 2277-88.

7. Tang SC, Lai KN. The pathogenic role of the renal proximal tubular cell in diabetic nephropathy. Nephrology, dialysis, transplantation : official publication of the European Dialysis and Transplant Association European Renal Association. 2012; 27: 3049-56.

8. Nieto MA, Huang RY, Jackson RA, Thiery JP. Emt: 2016. Cell. 2016; 166: 21-45.

9. Zeisberg M, Duffield JS. Resolved: EMT produces fibroblasts in the kidney. Journal of the American Society of Nephrology : JASN. 2010; 21: 1247-53.

10. Wang JY, Gao YB, Zhang N, Zou DW, Wang P, Zhu ZY, et al. miR-21 overexpression enhances TGF-beta1-induced epithelial-to-mesenchymal transition by target smad7 and aggravates renal damage in diabetic nephropathy. Molecular and cellular endocrinology. 2014; 392: 163-72.

11. Rivera-Barahona A, Perez B, Richard E, Desviat LR. Role of miRNAs in human disease and inborn errors of metabolism. Journal of inherited metabolic disease. 2017; 40: 471-80.

12. Kolling M, Kaucsar T, Schauerte C, Hubner A, Dettling A, Park JK, et al. Therapeutic miR-21 Silencing Ameliorates Diabetic Kidney Disease in Mice. Molecular therapy : the journal of the American Society of Gene Therapy. 2017; 25: 165-80.

13. Pearlman RL, Montes de Oca MK, Pal HC, Afaq F. Potential therapeutic targets of epithelial-mesenchymal transition in melanoma. Cancer letters. 2017; 391: 125-40.

14. Bugide S, Gonugunta VK, Penugurti V, Malisetty VL, Vadlamudi RK, Manavathi B. HPIP promotes epithelial-mesenchymal transition and cisplatin resistance in ovarian cancer cells through PI3K/AKT pathway activation. Cellular oncology (Dordrecht). 2017; 40: 133-44.

15. Liu H, Wang X, Liu S, Li H, Yuan X, Feng B, et al. Effects and mechanism of miR-23b on glucose-mediated epithelial-to-mesenchymal transition in diabetic nephropathy. The international journal of biochemistry \& cell biology. 2016; 70: 149-60.

16. Jiang $\mathrm{BH}$, Liu LZ. PI3K/PTEN signaling in angiogenesis and tumorigenesis. Advances in cancer research. 2009; 102: 19-65.

17. Chang RM, Xu JF, Fang F, Yang H, Yang LY. MicroRNA-130b promotes proliferation and EMT-induced metastasis via PTEN/p-AKT/HIF-1alpha signaling. Tumour biology : the journal of the International Society for Oncodevelopmental Biology and Medicine. 2016; 37: 10609-19.

18. Yuan J, Benway CJ, Bagley J, Iacomini J. MicroRNA-494 promotes cyclosporine-induced nephrotoxicity and epithelial to mesenchymal transition by inhibiting PTEN. American journal of transplantation : official journal of the American Society of Transplantation and the American Society of Transplant Surgeons. 2015; 15: 1682-91.

19. Ge Y, Xie H, Li S, Jin B, Hou J, Zhang H, et al. Treatment of diabetic nephropathy with Tripterygium wilfordii Hook F extract: a prospective, randomized, controlled clinical trial. J Transl Med. 2013; 11: 134

20. Zhu B, Wang Y, Jardine M, Jun M, Lv JC, Cass A, et al. Tripterygium preparations for the treatment of CKD: a systematic review and meta-analysis. American journal of kidney diseases : the official journal of the National Kidney Foundation. 2013; 62: 515-30.

21. Ma Z, Zhang X, Li L, Yang W, Wang S, Guo X, et al. Tripterygium Glycosides Tablet Ameliorates Renal Tubulointerstitial Fibrosis via the Toll-Like Receptor 4/Nuclear Factor Kappa B Signaling Pathway in High-Fat Diet Fed and Streptozotocin-Induced Diabetic Rats. Journal of diabetes research. 2015; 2015: 390428.

22. Gao Q, Shen W, Qin W, Zheng C, Zhang M, Zeng C, et al. Treatment of $\mathrm{db} / \mathrm{db}$ diabetic mice with triptolide: a novel therapy for diabetic nephropathy. Nephrology, dialysis, transplantation : official publication of the European Dialysis and Transplant Association - European Renal Association. 2010; 25: 3539-47.

23. Ma R, Liu L, Liu X, Wang Y, Jiang W, Xu L. Triptolide markedly attenuates albuminuria and podocyte injury in an animal model of diabetic nephropathy. Experimental and therapeutic medicine. 2013; 6: 649-56.

24. Guo H, Pan C, Chang B, Wu X, Guo J, Zhou Y, et al. Triptolide Improves Diabetic Nephropathy by Regulating Th Cell Balance and Macrophage Infiltration in Rat Models of Diabetic Nephropathy. Experimental and clinical endocrinology \& diabetes : official journal, German Society of Endocrinology [and] German Diabetes Association. 2016; 124: 389-98.

25. Cao Y, Huang X, Fan Y, Chen X. Protective Effect of Triptolide against Glomerular Mesangial Cell Proliferation and Glomerular Fibrosis in Rats Involves the TGF- beta 1/Smad Signaling Pathway. Evidence-based complementary and alternative medicine : eCAM. 2015; 2015: 814089.

26. Blantz RC, Singh P. Glomerular and tubular function in the diabetic kidney. Advances in chronic kidney disease. 2014; 21: 297-303.

27. Fioretto P, Barzon I, Mauer M. Is diabetic nephropathy reversible? Diabetes research and clinical practice. 2014; 104: 323-8.

28. Yuan XP, He XS, Wang CX, Liu LS, Fu Q. Triptolide attenuates renal interstitial fibrosis in rats with unilateral ureteral obstruction. Nephrology (Carlton, Vic). 2011; 16: 200-10.

29. Han F, Xue M, Chang Y, Li X, Yang Y, Sun B, et al. Triptolide Suppresses Glomerular Mesangial Cell Proliferation in Diabetic Nephropathy Is Associated with Inhibition of PDK1/Akt/mTOR Pathway. International journal of biological sciences. 2017; 13: 1266-75.

30. Hills CE, Squires PE. The role of TGF-beta and epithelial-to mesenchymal transition in diabetic nephropathy. Cytokine \& growth factor reviews. 2011; 22: 131-9.

31. Liu L, Salnikov AV, Bauer N, Aleksandrowicz E, Labsch S, Nwaeburu C, et al. Triptolide reverses hypoxia-induced epithelial-mesenchymal transition and stem-like features in pancreatic cancer by NF-kappaB downregulation. International journal of cancer. 2014; 134: 2489-503.

32. Liang Y, Jing Z, Deng H, Li Z, Zhuang Z, Wang S, et al. Soluble epoxide hydrolase inhibition ameliorates proteinuria-induced epithelial-mesenchymal transition by regulating the PI3K-Akt-GSK-3beta signaling pathway. Biochemical and biophysical research communications. 2015; 463: 70-5.

33. Zhang X, Liang D, Chi ZH, Chu Q, Zhao C, Ma RZ, et al. Effect of zinc on high glucose-induced epithelial-to-mesenchymal transition in renal tubular epithelial cells. International journal of molecular medicine. 2015; 35: 1747-54.

34. Paul P, Chakraborty A, Sarkar D, Langthasa M, Rahman M, Bari M, et al. Interplay between miRNAs and human diseases. Journal of cellular physiology. 2017.

35. Wu H, Kong L, Zhou S. The role of microRNAs in diabetic nephropathy. Journal of diabetes research. 2014; 2014: 920134

36. Zhao B, Li H, Liu J, Han P, Zhang C, Bai H, et al. MicroRNA-23b Targets Ras GTPase-Activating Protein SH3 Domain-Binding Protein 2 to Alleviate Fibrosis and Albuminuria in Diabetic Nephropathy. Journal of the American Society of Nephrology : JASN. 2016; 27: 2597-608.

37. Liu T, Nie F, Yang X, Wang X, Yuan Y, Lv Z, et al. MicroRNA-590 is an EMT-suppressive microRNA involved in the TGFbeta signaling pathway. Molecular medicine reports. 2015; 12: 7403-11.

38. Wang Y, Le Y, Xue JY, Zheng ZJ, Xue YM. Let-7d miRNA prevents TGF-beta1-induced EMT and renal fibrogenesis through regulation of HMGA2 expression. Biochemical and biophysical research communications. 2016; 479: 676-82.

39. Huang $\mathrm{Y}$, Tong J, He F, Yu X, Fan L, Hu J, et al. miR-141 regulates TGF-beta1-induced epithelial-mesenchymal transition through repression of HIPK2 expression in renal tubular epithelial cells. International journal of molecular medicine. 2015; 35: 311-8. 
40. Kang WL, Xu GS. Atrasentan increased the expression of klotho by mediating miR-199b-5p and prevented renal tubular injury in diabetic nephropathy. Scientific reports. 2016; 6: 19979.

41. Wang JY, Gao YB, Zhang N, Zou DW, Xu LP, Zhu ZY, et al. Tongxinluo ameliorates renal structure and function by regulating miR-21-induced epithelial-to-mesenchymal transition in diabetic nephropathy. American journal of physiology Renal physiology. 2014; 306: F486-95.

42. Ameres SL, Zamore PD. Diversifying microRNA sequence and function. Nature reviews Molecular cell biology. 2013; 14: 475-88.

43. Georgescu MM. PTEN Tumor Suppressor Network in PI3K-Akt Pathway Control. Genes \& cancer. 2010; 1: 1170-7.

44. He T, Guan X, Wang S, Xiao T, Yang K, Xu X, et al. Resveratrol prevents high glucose-induced epithelial-mesenchymal transition in renal tubular epithelial cells by inhibiting NADPH oxidase/ROS/ERK pathway. Molecular and cellular endocrinology. 2015; 402: 13-20. 